HNO 2018 $66: 878-879$

https://doi.org/10.1007/s00106-018-0579-1

(c) Springer Medizin Verlag $\mathrm{GmbH}$, ein Teil von Springer Nature 2018

CrossMark

\section{T. K. Hoffmann}

Klinik für Hals-, Nasen-, Ohrenheilkunde, Kopf- und Halschirurgie, Universitätsklinik Ulm, Ulm, Deutschland

\title{
Aktuelle Therapiekonzepte bei Kopf-Hals-Karzinomen
}

\section{Highlights des ASCO-Meetings 2018}

Alle Jahre wieder findet der Kongress der American Society of Clinical Oncology (ASCO) in Chicago statt. Der mit über 40.000 Teilnehmern weltweit größte internationale Kongress ist ein wichtiger Impulsgeber für die Diagnostik und Therapie von Krebspatienten. Längst ist Multimodalität und Interdisziplinarität der klinische Standard bei fortgeschrittenen Kopf-Hals-Karzinomen, was auch bei der Vorstellung verschiedener klinischerStudien verdeutlicht wird. Inzwischen hat diese Tumorentität das ursprüngliche Nischendasein verlassen und wird diagnostisch wie auch therapeutisch durch eine hohe Spezialisierung der Experten u.a. aus den Bereichen Kopf-Hals-Chirurgie, Strahlentherapie und Onkologie geprägt.

\section{》) Multimodalität und Interdisziplinarität sind klinischer Standard bei fortgeschrittenen Kopf-Hals-Karzinomen}

In den folgenden Übersichtsartikeln werden die wichtigsten Studienergebnisse zur Behandlung von Kopf-Hals-Malignomen zusammengefasst und dabei kritisch in den Kontext der Studienresultate der vergangenen Jahre eingeordnet.

\section{Chemotherapie}

Der bisherige systemtherapeutische Erstlinienstandard in der Palliation von Patienten mit Plattenepithelkarzinomen des Kopf-Hals-Bereichs ist seit einer Dekade das sog. EXTREME-Schema, bestehend aus Cisplatin, 5-Fluorouracil (5-FU) und Cetuximab, welches aber nicht immer effektiv und auch nebenwirkungsbehaftet ist. Dieser Standard unterliegt aktuell vergleichenden Untersuchungen beispielsweise i. R. einer Studie von Friesland et al., welche bei Erhalt von (Carbo-)Platin und Cetuximab das 5-FU durch Paclitaxel ersetzt und bei gleicher Wirksamkeit ein günstigeres Nebenwirkungsprofil erzielt [4]. Die Kombination von Palbociclib (Zellzyklusinhibition) und Cetuximab zeigte bei für humanes Papillomavirus (HPV-)negativen platinprogredienten Kopf-Hals-Karzinomen eine gute Ansprechrate bei akzeptabler Tolerabilität [1], wobei eine Testung gegen den aktuellen Standard (z. B. Nivolumab) in weiterführenden Studien erfolgen muss.

\section{Immuntherapie}

Nach Jahren der Stagnation gehört die Immuntherapie inzwischen $\mathrm{zu}$ den dynamischsten Entwicklungsfeldern in der Kopf-Hals-Onkologie. Nicht nur aufgrund der immunsuppressiven Eigenschaften der Kopf-Hals-Karzinome, sondern auch wegen deren verhältnismäßig hoher Immunogenität ist diese Tumorentität in den Fokus entsprechender Bemühungen geraten. Ein Durchbruch ergab sich durch Zulassung von Nivolumab bei Patienten mit einem platinrefraktären und rekurrenten Tumor, wobei offensichtlich auch die Älteren $\mathrm{zu}$ profitieren scheinen [5]. Zudem werden aktuell verschiedene Checkpoint-interagierende Antikörper (z.B. OX 40) in unterschiedlichen Settings (palliativ, neoadjuvant, adjuvant) unter Berücksichtigung von Lebensqualität und Nebenwirkungsprofil untersucht. Schließlich wurden Phase-I/II-Studien zur Kombination des Checkpointinhibitors Pembrolizumab mit neuartigen Zielstrukturen (Multikinase-Inhibitor, Histon-Deacetylase-Inhibitor, genetisch verändertes onkolytisches Herpes-simplex-Virus) vorgestellt, wobei praxisrelevante Phase-III-Studien zu immuntherapeutischen Substanzen diesjährig nicht präsentiert wurden.

\section{Humanes Papillomavirus}

Nicht zuletzt durch die 8. Ausgabe der TNM-Klassifikation im letzten Jahr ist die hohe prognostische Relevanz einer HPV-Infektion für Patienten mit Plattenepithelkarzinomen des Oropharynx international sichtbar geworden. Zwangsläufig sind Deeskalationsstudien die Folge, welche die Möglichkeit eines günstigen Nebenwirkungsprofils bei gleicher Wirksamkeit explorieren, was gerade bei den vielfach jungen Betroffenen zwecks Vermeidung der Spättoxizität einer Radiochemotherapie eine Rolle spielt.

》) Deeskalationsstudien
explorieren die Möglichkeit eines
günstigen Nebenwirkungsprofils
bei gleicher Wirksamkeit

War man lange davon ausgegangen, dass v. a. eine bessere Strahlensensitivität der HPV-positiven Tumoren für die günstige Prognose verantwortlich ist, so zeigt die retrospektive Studie von Cramer et al., dass die alleinige Chirurgie bei frühen Tumorstadien (R0-resezierte HPV-positive Oropharynxkarzinome im Stadium I/II, maximal 4 positive 
Lymphknoten, mikroskopisch extranodale Extension) ausreichend zu sein scheint [2]. Weitere Studien beschäftigen sich aktuell mit der Betrachtung anderer Tumorlokalisationen und der Frage nach der Zuverlässigkeit/Sinnhaftigkeit der alleinigen Detektion von p16, welche eine, wenn auch geringe, diagnostische Lücke offen lässt. Auch wenn in $\mathrm{Zu}$ kunft eine Deeskalation bei bestimmten Formen des HPV-induzierten Oropharynxkarzinoms wahrscheinlich ist, so ist eine generelle Deeskalation nach der aktuellen Datenlage immer noch nicht indiziert.

\section{Strahlentherapeutische Studien}

Bezug auf Oropharynxkarzinome nimmt auch die „Best-of-Studie“, welche die transorale Chirurgie bei Tumoren der Kategorie cT1/T2 cNo mit einer definitiven Strahlentherapie vergleicht und deren Rekrutierungsphase auch in einigen deutschen Tumorzentren begonnen hat [6]. Ein Schwerpunkt der strahlentherapeutischen Bemühungen war die Kombination etablierter RadiochemotherapieKombinationen (Cisplatin, Cetuximab) und „neuen“ (Checkpointinhibitoren) Antikörpern in der Kombination mit einer Strahlen(chemo)therapie, ohne dass sich hieraus unmittelbar Handlungskonsequenzen ableiten ließen [3]. Eine erste systematische Evaluation des abskopalen Effekts einer Radiotherapie zur Immuntherapie hat in einer kleinen Patientenkohorte leider keinen positiven Effekt ergeben, was möglicherweise auf gegenregulatorische Immuneffekte zurückzuführen ist.

\section{Schilddrüsenmalignome}

Als Kopf- und Halschirurgen haben wir zwangsläufig Berührungspunkte mit der Schilddrüse und deren Tumoren, weshalb ein separates Kapitel in den Sonderband integriert wurde. Auch wenn zur zweifelsohne wichtigen Chirurgie der Schilddrüsenmalignome keine wegweisenden Studien präsentiert wurden, gibt es multizentrische Beobachtungen zum praktischen Einsatz von TyrosinkinaseInhibitoren beim radioiodrefraktären differenzierten Schilddrüsenkarzinom.
Außerdem scheinen die prognostisch ausgesprochen schlechten anaplastischen Schilddrüsenkarzinome von einer individualisierten multimodalen Therapie zu profitieren.

Insgesamt ist der Kongress ein wichtiger Impulsgeber für die Behandlung der uns anvertrauten Patienten, und wir hoffen, Ihnen mit den Artikeln eine gute Übersicht über das sich stetig wandelnde Feld der Kopf-Hals-Onkologie geben zu können.

Eine interessante Lektüre wünscht Ihnen

Ihr

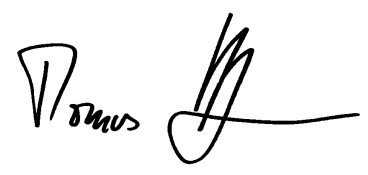

Prof. Dr. Thomas Hoffmann

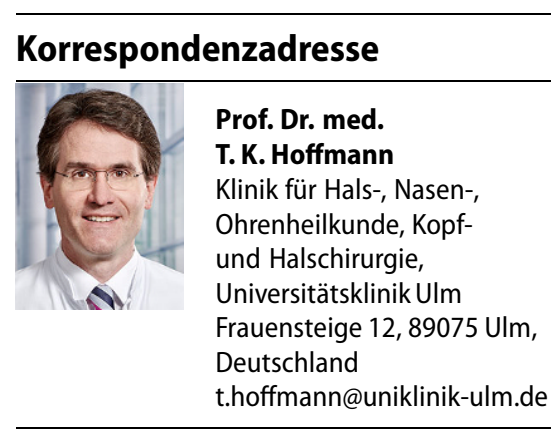

Interessenkonflikt. T.K. Hoffmann gibt an, dass kein Interessenkonflikt besteht.

\section{Literatur}

1. Adkins D, Oppelt PJ, Ley JC et al (2018) Multicenter phase II trial of palbociclib, a selective cyclin dependent kinase (CDK) 4/6 inhibitor, and cetuximab in platinum-resistant HPV unrelated (-) recurrent/metastatichead and necksquamous cell carcinoma (RM HNSCC). JClin Oncol 36:6008-6008

2. Cramer JD, Ferris RL, Duvvuri U (2018) Treatment deintensification to surgery only for stage I human papillomavirus-associated oropharyngeal cancer. JClin Oncol 36:6003-6003

3. Ferris RL, Gillison ML, Harris J et al (2018) Safety evaluation of nivolumab (Nivo) concomitant with cetuximab-radiotherapy for intermediate (IR) and high-risk (HR) local-regionally advanced head and neck squamous cell carcinoma (HNSCC): RTOG 3504. JClin Oncol 36:6010-6010

4. Friesland S, Tsakonas G, Kristensen C et al (2018) Randomised phase II study with cetuximab in combination with 5-FU and cisplatin or carboplatin versus cetuximab in combination with paclitaxel and carboplatin for treatment of patients with relapsed or metastatic squamous cell carcinoma of the head and neck (CETMET trial). J Clin Oncol 36:6032-6032

5. Saba NF, Blumenschein GR, Guigay J et al (2018) Nivolumab (nivo) vs investigator's choice (IC) in patients (pts) with recurrent or metastatic (R/M) squamous cell carcinoma of the head and neck (SCCHN): Analysis of CheckMate 141 by age. J Clin Oncol 36:6028-6028

6. Simon C, Caballero CA, Fortpied C et al (2018) BEST OF: A phase III study assessing the best of radiotherapy (Intensity Modulated RadioTherapy, IMRT) compared to the best of surgery (TransOral Surgery, TOS) in patients with T1-T2, N0 oropharyngeal squamous cell carcinoma (OPSCC). JClin Oncol 36:TPS6098-TPS6098 